\title{
Resection or Preservation? A Tough Surgical Decision for Chinese Breast Cancer Patients Who are in Line with Breast Conserving
}

Pingting Zhu ( $\sim$ ptzhu@yzu.edu.cn )

Yangzhou University https://orcid.org/0000-0002-1826-2725

Qiaoying Ji

Yangzhou University

Xinyi Liu

Yangzhou University

Ting Xu

Yangzhou University

Qiwei Wu

Yangzhou University

Yuejuan Wang

Yangzhou University

\section{Research Article}

Keywords: breast cancer, decision-making, surgery, Chinese, qualitative

Posted Date: November 23rd, 2021

DOI: https://doi.org/10.21203/rs.3.rs-571616/v2

License: (c) (i) This work is licensed under a Creative Commons Attribution 4.0 International License.

Read Full License 


\section{Abstract \\ Purpose}

Breast cancer patients who are in line with breast conserving are faced more challenges in making surgery decision, because breast conservation and mastectomy have equivalent oncologic outcomes. The purpose of this study is to analyze the surgical decision-making process for breast cancer patients and further explore the reasons why do Chinese women who are in line with breast conserving are more likely to choose mastectomy.

\section{Methods}

A qualitative study was conducted. With the sample saturation principle, data collected by semistructured interviews with 24 breast cancer patients who are in line with breast conserving. Colaizzi's method of phenomenology was used for data analysis.

\section{Results}

Three major themes emerged from the data. The themes were the following: (1) lack of significant decision-making support (stereotyping of breast cancer surgery, inferior quality of interaction with health professions, and without the help of decision aids); (2) can't mull under the strike (immediacy of the decision, breasts are out of deliberating, and escape); and (3) edified by the Chinese culture (deep family values, hiding concerns about femininity, and conservative character).

\section{Conclusions}

Our findings explore the reasons why more Chinese breast cancer patients with breast conserving conditions choose mastectomy, and help patients to choose knowledge-based treatment options which are concordant with their needs, values, and preferences.

\section{Background}

Breast cancer has become the most diagnosed cancer in the world.[1] In China, the rete of breast cancer is on the rise and 59.0/10 million in 2020, and ranking the incidence rate of female malignant tumors.[2, 3] At present, breast-conserving surgery and mastectomy are the main surgical methods for breast cancer patients.[4] Studies have shown that breast conserving surgery with radiation and mastectomy are equally effective surgical treatment options for early-stage breast cancer,[5] but the loss of breast will show the loss of self-esteem and a sense of diminished femininity. ${ }^{[6]}$ Like the NIH Consensus conference of 1990 concluded that, "breast conservation treatment... is preferable because it provides survival equivalent to total mastectomy... while preserving the breast". And breast has long been given the status 
of infinite honor and admiration by Chinese people. In the novel Big Breasts and Wide Hips written by Nobel Prize winner Mo Yan, breast is not only the symbol of female fertility, but also the origin of human life.[7] In addition, some Chinese think that the breast is the symbol of feminine charm and sex.[8, 9] Therefore, considering the high status of breast in Chinese culture and the fact that breast conserving surgery has more benefits than mastectomy, $[10,11]$ it might be inferred that women who are in line with breast conserving are more likely to choose breast conserving surgery. However, even in the early stage of breast cancer, the probability of Chinese breast cancer patients choosing breast conserving surgery is still relatively low.[12] Data show that the breast conserving rate of breast cancer in China is only about $11.88 \%$, while the breast conserving rate in the United States is as high as $65.5 \%$ in the early stage of breast cancer. $[13,14]$ Why do Chinese women who are in line with breast conserving choose radical mastectomy more? It must be a tough decision-making process to choose breast resection or breast preservation for Chinese women.

Worldwide, it has been observed great differences in the choice rate of breast conserving surgery.[15, 16] Some quantitative studies have shown that fear of cancer recurrence,[17] concerns about radiation,[18] and demographic factors such as age and education are factors that affect the choice of surgery.[19] However, quantitative studies are unable to describe why women are making this choice.[20] In order to further reveal the surgical decision-making process for Chinese breast cancer patients and understand why do Chinese women who are in line with breast conserving surgery are more likely to choose mastectomy, we conducted a qualitative study on patients who met the conditions of breast conserving but chose mastectomy.

\section{Methods}

\section{Design}

This qualitative study was conducted using in-depth, semi-structured, and face-to-face methods to analyze the surgical decision-making process for breast cancer patients and explore the reasons why do Chinese women are more likely to choose mastectomy.

\section{Participants}

Purposive sampling was used to extraction breast cancer patients with surgical decision-making experience according to the maximum difference sampling strategy. The inclusion criteria for participants consisted of the followings: (1) Be over the age of 18 years and in line with breast conserving surgery; (2) Experienced surgical decision making; (3) With normal cognitive ability and language expression ability, no verbal communication barrier; (4) Informed of the purpose and content of this study and voluntary participation. The exclusion criteria were inability to complete the interview. Data collection continued until information was saturated and no new themes or information emerged from the interviews. Data saturation was found to be achievable with 24 patients $(N=24)$. Participants' demographic characteristics were shown in Table 1. 
Table 1

Demographic Characteristic of the

Participants $(\mathrm{N}=24)$

\begin{tabular}{|ll|}
\hline Demographic data & $\mathrm{n}(\%)$ \\
\hline Age & \\
\hline $45-59$ & $7(29 \%)$ \\
$\geq 60$ & $11(46 \%)$ \\
\hline Education level & $6(25 \%)$ \\
\hline Illiterate & $2(8 \%)$ \\
\hline Primary & $3(13 \%)$ \\
\hline Secondary & $7(29 \%)$ \\
\hline Higher Secondary & $6(25 \%)$ \\
\hline Honors/Degree & $4(17 \%)$ \\
\hline Masters & $2(8 \%)$ \\
\hline Marital status & \\
\hline Married & $20(83 \%)$ \\
Separated/widowed & $4(17 \%)$ \\
\hline Children & \\
\hline Yes & $23(96 \%)$ \\
No & $1(4 \%)$ \\
\hline
\end{tabular}

\section{Data collection}

Data were collected through semi-structured interviews from March to August in 2021. Face-to-face interviews were conducted by two researchers in a private room in the hospital, and after interviews the researcher returns the fundamental structure statement to all participants to ask whether it captures their experience.[21] The semi-structured interview consisted of open-ended questions (Table 2). Interviews were audio-recorded with permission and using two recording devices for quality assurance. Interviews lasted 22 to 74 min (median $45 \mathrm{~min}$ ). 
Table 2

Interview guide

\begin{tabular}{|ll|}
\hline Number & Questions \\
\hline Q1 & $\begin{array}{l}\text { How did you feel when you were diagnosed with breast cancer, both physically and } \\
\text { mentally? }\end{array}$ \\
\hline Q2 & Could you describe your surgical decision-making process in detail? \\
\hline Q3 & What are the factors that influenced your decision? \\
\hline Q4 & What resources have you drawn to cope with your decision-making? \\
\hline Q5 & Could you tell how do you feel about the surgery? \\
\hline Q6 & Is there anything you want to talk about that I didn't ask? \\
\hline
\end{tabular}

\section{Data analysis}

Data of the interview were transcribed within 24 hours. Employed the

Colaizzi's phenomenological analysis method,[22, 23] which can provides a clear structure for data analysis. And in strict accordance with the requirements of the method of data system analysis. Two researchers independently read each transcribed text, extracted and encoded the statement. Then they classified those codes into themes and sub-themes and had regular and continuing discussion to verify the appropriateness of the conceptual meanings and terminology.

\section{Ethical considerations}

The proposal of this study was approved by the Ethics Committee of School of

Nursing of Yangzhou University, with the IR code: YZUHL2021006. Verbal and/or written informed consent was obtained from all participants. We will have the guarantee about the principles of confidentiality and anonymity, and the freedom to withdraw from the study at any time without any consequences.

\section{Result}

\section{Theme1 Lack of significant decision-making support}

Stereotyping of breast cancer surgery Patient's own knowledge reserve can support them to make surgical decisions in the best interests, but fewer than half of women undergoing mastectomy have adequate knowledge and skills to make the right surgical decision. They rigidly associate breast cancer with radical mastectomy, and have deep-rooted misconceptions about the surgery of breast cancer.

P6 Got breast cancer must be removed, there is no other way, had to do mastectomy, only this way! 
P1 Breast removal is life saving! The breasts left is like a time bomb, and it will recur. It's better to take it off and cut it off, so it won't recur.

P17 There will be no sequelae after mastectomy. It's the safest choice to cut it off!

Unequal knowledge with health professionals Health professional support plays an important role in the surgical decision-making process for Chinese patients who identify with a system that values professional opinions. Health professionals are regarded as authority, and Chinese patient allegiance to authority which result in they respecting and accepting the advice put forward by health professionals. However, due to the knowledge gap between health workers and patients, as well as the lack of expression ability of health workers, patients in our study $(n=18)$ reported a bit of variability in their interactions with physicians. Some women may feel they are being directed to choose radical mastectomy, or if their decision has already been made, feel that the physician has provided tacit approval or agreement. In addition, health professions may have projection bias in the process of decision-making, which means that the provider influences surgical decision making with his/her preferences. This makes it difficult for women to make surgical decisions that maximize their preferences.

P4 The director is an expert in breast cancer, is the authority. I believe in doctors, and listen to them!

P7 My doctor said a lot, but I didn't understand, so I told the doctor that it's all up to you.

P19 My condition allowed me to have breast-conserving surgery, but I think the doctor's idea was that radical surgery was better. Their experience told me that it is better to choose radical operation.

Without the help of decision aids Women always enlisting the doctor's opinion as the main driving force of treatment decisions, but then solidifying this decision by or opinions from other sources. Generally, women tend to rely on a variety of online sources to obtain information about this process, many of which contain inaccurate or incomplete information. Therefore, they need standardized educational tools such as decision aids that aim to provide information to facilitate better decision making, taking the patient's own values and preferences into account.

P15 I searched for breast cancer related information on the Internet, but the information on the Internet was a lot of messy, which often misled me.

P6 I don't have a brochure or video from health workers about how to choose surgery.

P18 Sometimes I look up the information about breast cancer surgery on the search engine, but most of the information is obtained in the process of communicating with patients.

\section{Theme 2 Can't mull under the strike}


Immediacy of the decision Due to the limitation of medical resources, decisions regarding the choice of procedure type must often be made shortly after diagnosis, and most patients express they wanted to move to treatment as quickly as possible. But in a short period of time, participants had no established relationship with most of health workers. At the same time, they were dealing with the reality of being ill and learning a new medical vocabulary. So they don't have a lot of time to think deeply and analyze their own situation.

P21 When I knew I had breast cancer, I thought the hospital had made a mistake. I couldn't accept the fact that I had breast cancer, I broke down, I collapsed! On the day when the results came out, the doctor would make a preliminary treatment process, and on the third day I had an operation. At that time, I didn't have the ability to think too much and the quickest solution I could think of was to remove the breast.

P10 The doctor calmly said that I needed an operation and asked me if I wanted to choose breast conserving surgery. I immediately felt like it was spinning, very dizzy. I asked my doctor, "do you mean I have cancer? How come it's time to discuss whether to choose breast-conserving surgery? "

Breasts are out of deliberating When they get the diagnosis of the disease, under the information bombardment, their first thought is their longevity and family. They can't process quantities of information quickly and think about their own needs. Young women with children worry about their life expectancy and question whether they can see their children grow up to be independent. The elderly women are worried about their health and don't want to bring burden to their families due to the deterioration of their health.

P19 At that time, I just thought that life was more important than anything! If health can be achieved by removing a breast, it's very cost-effective. After all, I have two daughters, the younger one is only a few months old.

P6 The first thing I thought about was my family when I decided to have an operation. Breast conserving surgery has a higher recurrence rate and requires a longer treatment period. I wanted to recover as soon as possible and fulfilled my family responsibilities.

Escape Breast cancer patients often feel very fearful and stressed when they get their cancer diagnosis. At the same time, they get too much information about breast cancer in a short time. When they don't know what to do in the face of disease, they will choose to escape. Women may tend to take passive roles in treatment decision-making and delegated decision-making to their health professions or family. But for health professions and their family, they pay more attention to curative effect and economic benefit of treatment, survival is usually more important than quality of life in traditional Chinese culture. Therefore, there may be inconsistencies between actual and preferred value.

P3 I know a little bit about breast cancer, but my family knows. I didn't make a choice in the surgical decision-making process. My husband made a choice for me. 
P22 I didn't ask, I didn't want to ask, I was tired of it. The less I knew, the better. The more I knew, the more I though, I couldn't sleep at night.

\section{Theme 3 Edified by the Chinese culture}

Deep family values Culture can influence how individuals respond to cancer diagnosis and treatment. For Chinese people the collective concept of family

is deep-rooted and far-reaching, and they face major events with family as a unit, the body is not only his or her own but also that of the whole family. Therefore, the diagnosis of cancer is not only an individual event, but also a family crisis. Family accompanied patients to medical visits and act as a medium of communication medium between health professions and patients. In the process of making decisions, women tend to give up his or her personal appeal and compromise with family members to reach a common decision.

P13 To be exact, the disease is not on me, but on our family. It's a family responsibility.

P7 I wanted to have breast conserving surgery, but my grandchildren wouldn't let me. They are very strong advocates of getting mastectomy. They persuaded me with their though and all.

P8 Treatment is painful, but it should be treated positively. After all, there is a family. There are so many people in this world that it doesn't matter if I leave this world. But for a family, if the elders are there, the family will be cohesive. There are too many people in the world, but there are very few in your family.

Hiding concerns about femininity Chinese cultural philosophies, which are completely different from western cultural foundations, shape individual perceptions of disease. Influenced by traditional culture, Chinese people will to hiding their concerns about femininity to avoid disturbing the harmonious equilibrium of interpersonal relationships. In addition, in Chinese traditional culture "survival" is a priority over everything, but the choice of breast conserving surgery is considered "for beauty, not for survival". Especially when other patients choose mastectomy, but participants express that they prefer breast preservation, the surrounding people may comment unfriendly on participants. Therefore, many women will hide their desire for breast and choose radical mastectomy to avoid unfriendly comments from people around them.

P3 Although I wanted to choose breast-conserving surgery, my husband and mother didn't agree. I didn't tell them what I thought because I didn't want to burden them. Many words can only be buried in their own heart,sometimes I feel like I'm not me when I'm sick.

P16 I felt that I aged, breast cancer patients younger than me had their breasts removed, so it would be very strange if I didn't remove my breasts. If I showed that I wanted to keep my breasts, others might whisper bad things about me. 
Conservative character Some of the old Chinese sayings, such as "stop the boiling by taking off the fire strike at the root of the trouble" and "get rid of the weeds by killing the root", affect the choice of breast cancer patients. They believe that breast cancer should be treated with caution and the source of trouble should be removed thoroughly to prevent future problems. To be on the safe side, they prefer mastectomy to reduce the risk of recurrence.

P14 The recurrence rate of breast conserving surgery was high. If it was not completely removed, it might have to be operated again later, I didn't want to take chances! In order to avoid the follow-up trouble, I chose mastectomy.

P9 I didn't know if my cancer cells had gone to other parts of my breast, lumpectomy might not be able to clean up the cancer cells. I finally decided to have a mastectomy. It's safer, just to make sure all the cancer cells were removed.

\section{Discussion}

In this study, we used qualitative research methods to investigate the details of surgical decision-making process of Chinese breast cancer survivors, and provided insight into the thoughts and viewpoints of Chinese breast cancer survivors who chose mastectomy but met the requirements of breast conserving. For Chinese breast cancer patients, the decision-making process is not only a process of making their own choices, but also influenced by health professionals, family members and cultural background.

Previous studies have shown that health professionals are regarded as authoritative in Chinese culture and Chinese patients with breast cancer often depend on health professionals to make medical decisions. $[24,25]$ But in the decision-making process of surgery, physicians expressed more limited considerations, emphasize the efficacy, side effects and cost of treatment,[26] rather than giving priority to the patient's own will. Even though health professionals believe that the patient's goals are important, they often make assumptions about preferences rather than asking the patient explicitly. But the decisionmaking process of breast cancer surgery are unique, because surgical options-breast conservation and mastectomy-have equivalent oncologic outcomes.[27] Therefore, providers should avoid projection bias in which the provider influences surgical decision making with her preferences. Patient preference should be honored when there are medically equivalent treatment options, integrating the physician's professional expertise with patient values and expectations.[28] In addition, Chinese obedience to authority makes women don't dare to ask health professions directly for fear of asking silly questions or being labeled as a "bad patient".[29] Women may prefer health professional to provide clear information without ask to many questions.[30] Therefor, health professions need to discuss the practical, longer-term real-world challenges associated with each of the surgical treatment options with patients. And Surgical consultation needs to be discussed to assessing how risk is interpreted and defined by each patient as well as understanding patients' view of their body and breasts. The personal circumstances of patients should be included, and encourage women to reflect on their personal values and surgical preferences for his personal situation. In addition, women in our interview also mentioned that they can't think about all 
kinds of problems in their limited time from being told the diagnosis of the disease to making surgical decisions. And health professionals as a reliable source of patient information, should give the patient as much time to think as the condition and save patients from the information bombing. Although women always enlisting the health professions' opinion as the main driving force of treatment decisions, they also look for other channels to help them make surgical decisions.[31] However, due to the variety of information on the Internet, it is difficult to distinguish the authenticity of information for breast cancer patients without medical basis. In our interview, the interviewees had not access decision-making aids. For introverted Chinese patients, decision-making aids may be an effective tool to help breast cancer patients who need to make surgical decisions to improving self-reported satisfaction with information and patients' perceived involvement in the decision-making process.[32]

The treatment of cancer is rarely just a matter of patients, the function of patient family unit is very important in the process of decision-making, this is consistent with study of Wang, L., et al.[9] Family members of Chinese breast cancer patients usually actively participate in the treatment decision of breast cancer patients. They accompanied patients to medical visits, as a medium of communication between health professions and patients, and even replace the patients who are at a loss under the impact to make surgical decisions. However, family members of breast cancer patients who have not received training in medical interpretation may not be able to accurately convey information to women. And we notice that in order not to disturb or burden their family, breast cancer patients often chose to hide their expectations to meeting the requirements of family members.[33, 34] In Chinese culture, women take their family responsibility very seriously, they tend to fulfill their expected social roles. But breast cancer makes women dependent on others, and family responsibility needs to be transferred to family members during treatment period, which was also reported by the study of Fu et al.[35] In order to avoid the disorder within the family, they hope to cure the disease as soon as possible by removing the breast. Thus, consider family and cultural influences, health professionals ought to appropriate psychological interventions that cognizant of patients' emotional reaction to breast cancer.

In addition, the treatment decisions of many breast cancer patients will be affected by the medical environment.[36] In China patients' perceptions and the medical environment, mastectomies are actually very common, while breast conserving surgery is rare. Patients' decision-making on breast cancer surgery is affected by their prior knowledge.[37] When all the patients around choose mastectomy, they will also be more inclined to choose mastectomy. The cautious character of the Chinese also prompted them to choose mastectomy to reduce the risk of recurrence of breast cancer, they said they do not want to live in the shadow of breast cancer in the future, which is consistent with the results of Rosenberg, S.M., et al. [38]

\section{Limitations}

Our research has some limitations. Our participants were mainly from Yangzhou, a third-tier city in China. And they had made their surgical decision before being interviewed, the data are affected by recall and memory bias. Future research should include patients who are making surgical decisions. 


\section{Conclusion}

Our findings analyze the surgical decision-making process for breast cancer patients and further reveal why Chinese breast cancer patients who are in line with breast conserving surgery are more likely to choose mastectomy. The main reasons for women to choose mastectomy are: lack of decision-making support, unable to deliberate in the strike, and edified by the Chinese culture. We hope that these findings will help to guide patients to choose knowledge-based treatment options which are concordant with their needs, values, and preferences. And assist health care professionals to take care of women with Chinese cultural background in China and other countries.

\section{Declarations}

Funding: This study was funded by the National Natural Science Foundation of China (82174129), the Postgraduate Research \& Practice Innovation Program of Jiangsu Province (No.KYCX20_3006), Science Foundation of Nursing Research of Yangzhou University (HX2002) and Young Backbone Teacher Foundation of Yangzhou University.

Competing interests: The authors declare that they have no conflicts of interest.

Availability of data and material: N/A

Code availability: N/A

Authors' contributions: Concept-QYJ; design-PTZ, QYJ, XYL; interview-QYJ, XYL; data analysisPTZ,QYJ,XYL,TX,QWW,YJW; data interpretation-PTZ,QYJ,XYL,TX,QWW,YJW; first draft-QYJ; revisions and final manuscript-PTZ,QYJ,XYL,TX,QWW,YJW.

Ethics approval: This study was performed in line with the principles of the Declaration of Helsinki. Approval was granted by the Ethics Committee of School of Nursing of Yangzhou University, with the IR code: YZUHL2021006. Informed consent was taken prior to interview, including for use of anonymous quotes.

Consent to participate: N/A

Consent for publication: N/A

\section{References}

1. Sung H, Ferlay J, Siegel RL, Laversanne M, Soerjomataram I, Jemal A, Bray F (2021) Global cancer statistics 2020: GLOBOCAN estimates of incidence and mortality worldwide for 36 cancers in 185 countries. CA: a cancer journal for clinicians. https://doi.org/10.3322/caac.21660

2. Ferlay JEM, Lam F, Colombet M, (2021) Global Cancer Observatory:Cancer Today.https://gco.iarc.fr/today/fact-sheets-populations. Accessed Date, 2021 
3. (2020) [Guidelines for clinical diagnosis and treatment of advanced breast cancer in China (2020 Edition)]. Zhonghua zhong liu za zhi [Chinese journal of oncology] 42: 781-97. https://doi.org/10.3760/cma.j.cn112152-20200817-00747

4. Harbeck N, Penault-Llorca F, Cortes J, Gnant M, Houssami N, Poortmans P, Ruddy K, Tsang J, Cardoso F (2019) Breast cancer. Nature reviews Disease primers 5: 66. https://doi.org/10.1038/s41572-019-0111-2

5. Veronesi U, Cascinelli N, Mariani L, Greco M, Saccozzi R, Luini A, Aguilar M, Marubini E (2002) Twenty-year follow-up of a randomized study comparing breast-conserving surgery with radical mastectomy for early breast cancer. The New England journal of medicine 347: 1227-32. https://doi.org/10.1056/NEJMoa020989

6. Ashing-Giwa KT, Padilla G, Tejero J, Kraemer J, Wright K, Coscarelli A, Clayton S, Williams I, Hills D (2004) Understanding the breast cancer experience of women: a qualitative study of African American, Asian American, Latina and Caucasian cancer survivors. Psycho-oncology 13: 408-28. https://doi.org/10.1002/pon.750

7. Mo Y (2004) BIG BREASTS \& WIDE HIPS. Arcade New York

8. Zhai J, Weller-Newton JM, Shimoinaba K, Chen H, Copnell B (2020) Emerging From the "Ku:" Fluctuating in Adjusting With Breast Cancer-A Post-Traumatic Growth Theory Situated Within Chinese Culture. Qualitative Health Research 30: 1674-83.

https://doi.org/10.1177/1049732320930195

9. Wang L, Geng X, Ji L, Lu G, Lu Q (2020) Treatment decision-making, family influences, and cultural influences of Chinese breast cancer survivors: a qualitative study using an expressive writing method. Supportive care in cancer : official journal of the Multinational Association of Supportive Care in Cancer 28: 3259-66. https://doi.org/10.1007/s00520-019-05161-w

10. Howard-Anderson J, Ganz PA, Bower JE, Stanton AL (2012) Quality of life, fertility concerns, and behavioral health outcomes in younger breast cancer survivors: a systematic review. Journal of the National Cancer Institute 104: 386-405. https://doi.org/10.1093/jnci/djr541

11. Waks AG, Winer EP (2019) Breast Cancer Treatment: A Review. Jama 321: 288-300. https://doi.org/10.1001/jama.2018.19323

12. Fan L, Strasser-Weippl K, Li J-J, St Louis J, Finkelstein DM, Yu K-D, Chen W-Q, Shao Z-M, Goss PE (2014) Breast cancer in China. The Lancet Oncology 15: e279-e89. https://doi.org/10.1016/s14702045(13)70567-9

13. Kummerow KL, Du L, Penson DF, Shyr Y, Hooks MA (2015) Nationwide trends in mastectomy for early-stage breast cancer. JAMA surgery 150: 9-16. https://doi.org/10.1001/jamasurg.2014.2895

14. Zhang BL, Sivasubramaniam PG, Zhang Q, Wang J, Zhang B, Gao JD, Tang ZH, Chen GJ, Xie XM, Wang Z, Yang HJ, He JJ, Li H, Li JY, Fan JH, Wang X, Qiao YL (2015) Trends in Radical Surgical Treatment Methods for Breast Malignancies in China: A Multicenter 10-Year Retrospective Study. The oncologist 20: 1036-43. https://doi.org/10.1634/theoncologist.2014-0281 
15. Caldon LJ, Walters SJ, Reed JA, Murphy A, Worley A, Reed MW (2005) Case-mix fails to explain variation in mastectomy rates: management of screen-detected breast cancer in a UK region 19972003. British journal of cancer 92: 55-9. https://doi.org/10.1038/sj.bjc.6602264

16. Ishizaki T, Imanaka Y, Hirose M, Kuwabara K, Ogawa T, Harada Y (2002) A first look at variations in use of breast conserving surgery at five teaching hospitals in Japan. International journal for quality in health care : journal of the International Society for Quality in Health Care 14: 411-8. https://doi.org/10.1093/intqhc/14.5.411

17. Billig JI, Duncan A, Zhong L, Aliu O, Sears ED, Chung KC, Momoh AO (2018) The Cost of Contralateral Prophylactic Mastectomy in Women with Unilateral Breast Cancer. Plastic and reconstructive surgery 141: 1094-102. https://doi.org/10.1097/prs.0000000000004272

18. Caldon LJ, Collins KA, Wilde DJ, Ahmedzai SH, Noble TW, Stotter A, Sibbering DM, Holt S, Reed MW (2011) Why do hospital mastectomy rates vary? Differences in the decision-making experiences of women with breast cancer. British journal of cancer 104: 1551-7. https://doi.org/10.1038/bjc.2011.141

19. Gutnik L, Allen CM, Presson AP, Matsen CB (2020) Breast Cancer Surgery Decision Role Perceptions and Choice of Surgery. Annals of surgical oncology 27: 3623-32. https://doi.org/10.1245/s10434020-08485-8

20. Covelli AM, Baxter NN, Fitch MI, McCready DR, Wright FC (2015) 'Taking control of cancer': understanding women's choice for mastectomy. Annals of surgical oncology 22: 383-91. https://doi.org/10.1245/s10434-014-4033-7

21. Morrow R, Rodriguez A, King N (2015) Colaizzi's descriptive phenomenological method.

22. Edward KL, Welch T (2011) The extension of Colaizzi's method of phenomenological enquiry. Contemporary nurse 39: 163-71. https://doi.org/10.5172/conu.2011.163

23. Colaizzi P (1978) Psychological research as the phenomenologist views it.

24. Tam Ashing K, Padilla G, Tejero J, Kagawa-Singer M (2003) Understanding the breast cancer experience of Asian American women. Psycho-oncology 12: 38-58. https://doi.org/10.1002/pon.632

25. Xie B, Su Z, Liu Y, Wang M, Zhang M (2015) Health information wanted and obtained from doctors/nurses: a comparison of Chinese cancer patients and family caregivers. Supportive care in cancer : official journal of the Multinational Association of Supportive Care in Cancer 23: 2873-80. https://doi.org/10.1007/s00520-015-2651-7

26. Rocque GB, Rasool A, Williams BR, Wallace AS, Niranjan SJ, Halilova KI, Turkman YE, Ingram SA, Williams CP, Forero-Torres A, Smith T, Bhatia S, Knight SJ (2019) What Is Important When Making Treatment Decisions in Metastatic Breast Cancer? A Qualitative Analysis of Decision-Making in Patients and Oncologists. The oncologist 24: 1313-21. https://doi.org/10.1634/theoncologist.20180711

27. Throckmorton A, VanderWalde L, Brackett C, Dominici L, Eisenhauer T, Johnson N, Kong A, Ludwig K, O'Neill J, Pugliese M, Teller P, Sarantou T (2015) The Ethics of Breast Surgery. Annals of surgical oncology 22: 3191-6. https://doi.org/10.1245/s10434-015-4751-5 
28. Andersson GB, Chapman JR, Dekutoski MB, Dettori J, Fehlings MG, Fourney DR, Norvell D, Weinstein JN (2010) Do no harm: the balance of "beneficence" and "non-maleficence". Spine 35: S2-8. https://doi.org/10.1097/BRS.0b013e3181d9c5c5

29. Levesque JV, Gerges M, Wu VS, Girgis A (2020) Chinese-Australian women with breast cancer call for culturally appropriate information and improved communication with health professionals. Cancer reports (Hoboken, NJ) 3: e1218. https://doi.org/10.1002/cnr2.1218

30. Xie B, Su Z, Liu Y, Wang M, Zhang M (2017) Health information sources for different types of information used by Chinese patients with cancer and their family caregivers. Health expectations : an international journal of public participation in health care and health policy 20: 665-74. https://doi.org/10.1111/hex.12498

31. Kwok C, Koo FK (2017) Participation in treatment decision-making among Chinese-Australian women with breast cancer. Supportive care in cancer : official journal of the Multinational Association of Supportive Care in Cancer 25: 957-63. https://doi.org/10.1007/s00520-016-3487-5

32. Berlin NL, Tandon VJ, Hawley ST, Hamill JB, MacEachern MP, Lee CN, Wilkins EG (2019) Feasibility and Efficacy of Decision Aids to Improve Decision Making for Postmastectomy Breast Reconstruction: A Systematic Review and Meta-analysis. Medical decision making : an international journal of the Society for Medical Decision Making 39: 5-20.

https://doi.org/10.1177/0272989x18803879

33. Lu Q, Yeung NC, You J, Dai J (2016) Using expressive writing to explore thoughts and beliefs about cancer and treatment among Chinese American immigrant breast cancer survivors. Psycho-oncology 25: 1371-4. https://doi.org/10.1002/pon.3991

34. Warmoth K, Cheung B, You J, Yeung NCY, Lu Q (2017) Exploring the Social Needs and Challenges of Chinese American Immigrant Breast Cancer Survivors: a Qualitative Study Using an Expressive Writing Approach. International journal of behavioral medicine 24: 827-35. https://doi.org/10.1007/s12529-017-9661-4

35. Fu MR, Xu B, Liu Y, Haber J (2008) 'Making the best of it': Chinese women's experiences of adjusting to breast cancer diagnosis and treatment. Journal of advanced nursing 63: 155-65. https://doi.org/10.1111/j.1365-2648.2008.04647.x

36. Zhang T, Li H, Liu A, Wang H, Mei Y, Dou W (2018) Factors promoting resilience among breast cancer patients: a qualitative study. Contemporary nurse 54: 293-303.

https://doi.org/10.1080/10376178.2018.1502615

37. Kwok C, White K (2011) Cultural and linguistic isolation: the breast cancer experience of ChineseAustralian women - a qualitative study. Contemporary nurse 39: 85-94. https://doi.org/10.5172/conu.2011.39.1.85

38. Rosenberg SM, Greaney ML, Patenaude AF, Sepucha KR, Meyer ME, Partridge AH (2018) "I don't want to take chances.": A qualitative exploration of surgical decision making in young breast cancer survivors. Psycho-oncology 27: 1524-9. https://doi.org/10.1002/pon.4683 